\title{
Electrospun gelatin/PCL and collagen/PCL scaffolds for modulating responses of bone marrow endothelial progenitor cells
}

\author{
YANG HU ${ }^{1,2^{*}}$, BEI FENG ${ }^{3 *}$, WEIJIE ZHANG ${ }^{1,2}$, CHENXI YAN $^{1,2}$, QINKE YAO $^{1,2}$,

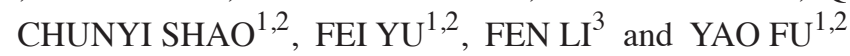 \\ ${ }^{1}$ Department of Ophthalmology, Ninth People's Hospital, Shanghai Jiao Tong University, School of Medicine; \\ ${ }^{2}$ Shanghai Key Laboratory of Orbital Disease and Ocular Oncology, Shanghai 200011; ${ }^{3}$ Department of Pediatric Cardiology, \\ Shanghai Children's Medical Center, Shanghai Jiao Tong University, School of Medicine, Shanghai 200127, P.R. China
}

Received March 22, 2018; Accepted September 13, 2018

DOI: $10.3892 / \mathrm{etm} .2019 .7387$

\begin{abstract}
The determination of potential transplantable substrates and substitution cells for corneal endothelium transplantation may compensate for the shortage of cornea donors. Appropriate biodegradable and biocompatible tissue-engineered substratum with seed cells for endothelial keratoplasty has been increasingly studied. In the present study, electrospun gelatin/polycaprolactone (PCL) and collagen/PCL scaffolds were successfully established. Bone marrow endothelial progenitor cells (BEPCs) were cultured on these scaffolds to determine whether the scaffolds may promote the proliferation of BEPCs as well as maintain stem cell characteristics. Two variations of hybrid scaffolds, collagen/PCL (70\% collagen and 30\% PCL) and gelatin/PCL (70\% gelatin and 30\% PCL), were established via electrospinning. Microscopic structure, hydrophilicity and wettability of the two scaffolds were subsequently investigated. BEPCs were separately cultured on the scaffolds and were also seeded on glass slides to establish the control group. Furthermore, cell
\end{abstract}

Correspondence to: Dr Yao Fu, Department of Ophthalmology, Ninth People's Hospital, Shanghai Jiao Tong University, School of Medicine, 639 Zhizaoju Road, Shanghai 200011, P.R. China

E-mail:drfuyao@126.com

Dr Fen Li, Department of Pediatric Cardiology, Shanghai Children's Medical Center, Shanghai Jiao Tong University, School of Medicine, 1678 Dong Fang Road, Shanghai 200127, P.R. China

E-mail: lifen@scmc.com.cn

*Contributed equally

Abbreviations: DPAI, 4,6-diamidino-2-phenylindole; PCL, polycaprolactone; AQP1, aquaporin 1; COL8A1, collagen type VIII alpha 1; ABCG2, ATP binding cassette subfamily G member 2; LGR5, leucine rich repeat containing $\mathrm{G}$ protein-coupled receptor 5; IL-1, interleukin 1

Key words: corneal endothelium, scaffolds, electrospun nanofibrous membrane, gelatin, collagen, bone marrow endothelial progenitor cell morphology; adherence, as determined by investigation of F-actin expression levels; proliferation, as determined via Cell Counting Kit-8 assays, Ki-67 staining and bromodeoxyuridine (BrdU) staining; and stem cell markers, as determined by cluster of differentiation (CD)-34 and CD-133 protein expression levels; were investigated. In addition, reverse transcription-quantitative polymerase chain reaction (RT-qPCR) was performed to determine gene expression. The two nanofiber scaffolds were established using electrospun techniques with expected hydrophilicity, wettability and biocompatibility. BEPCs were revealed to spread well on and strongly adhere to the collagen/PCL (70:30) and gelatin/PCL (70:30) scaffolds. Furthermore, Ki-67 and BrdU staining results revealed greater levels of positive dots on the two hybrid scaffolds compared with the control group. CD-34 and CD-133 protein staining demonstrated increased levels of fluorescence intensity on scaffolds compared with the control group. Furthermore, increased expression levels of differentiation markers, such as ATP binding cassette subfamily $\mathrm{G}$ member 2, leucine rich repeat containing $\mathrm{G}$ protein-coupled receptor 5 and CD166, were detected on both scaffolds. RT-qPCR results demonstrated that the expression of caspase- 3 , which is associated with apoptosis, was decreased on the two scaffolds compared with in the control group. The expression of inflammatory factors, including interleukin (IL)-1, exhibited a significant decrease on the gelatin/PCL scaffold compared with in the control group; whereas the difference between the expression level of IL-1 exhibited by the collagen/PCL group and the control group were not markedly different. Electrospun collagen/PCL and gelatin/PCL scaffolds exhibited the potential to enhance the adherence and proliferation of BEPCs. BEPCs cultured on the two scaffolds demonstrated increased stem cell characteristics and differentiation potential. Electrospun gelatin/PCL and collagen/PCL scaffolds may represent a promising substratum in tissue-engineered corneal endothelium.

\section{Introduction}

The human corneal endothelium represents the single cell layer between the corneal stroma and anterior chamber, which exhibits barrier and 'pump' functions to maintain corneal transparency (1). Trauma, diseases, surgery and aging may lead 
to a decrease in human corneal endothelial cells (HCECs) (2). Considering that HCECs do not proliferate in vivo, corneal transplantation is required for patients with corneal endothelial dysfunction in order to restore vision $(3,4)$. However, the shortage of cornea donors is problematic. Corneal surgeons may now replace injured corneal endothelia with a thin, transparent, biocompatible, tissue-engineered substratum exhibiting corneal endothelial cells or other replaceable cells (5). Tissue-engineered corneas are currently of interest, thus motivating researchers to develop an appropriate biocompatible scaffold and determine replaceable cells.

It has been recently demonstrated that nanofibrous structures formed by electrospinning may promote cell spreading, proliferation, migration and attachment (6,7). Nanofibrous scaffolds established by electrospinning may provide cells with a growth environment that is similar to extracellular matrices of native tissues. Nutrients and waste may be efficiently exchanged, and the large surface provided by electrospinning may contribute to the transduction of biochemical signals by cultured cells $(8,9)$. Natural polymers, such as collagen and gelatin, have been electrospun into fibrous scaffolds for cornea tissue engineering and endothelial cells (10-13). Gelatin and collagen are natural components of the extracellular matrix, which can provide an appropriate surface for cell proliferation, adhesion and differentiation (14). However, the mechanical characteristics of natural scaffolds are typically hard to establish. For example, when placed in liquid solution, natural scaffolds rarely retain their structural integrity $(15,16)$. However, the mechanical strength of synthetic electrospun scaffolds, such as polycaprolactone (PCL) and poly(L-lactide-co-glycolide), is usually satisfactory and their biodegradability is strongly regulated $(17,18)$. Scaffolds combining natural and synthetic polymers may simultaneously maintain the advantages and attenuate the disadvantages of scaffolding (19).

In our previous study $(20,21)$, it was indicated that bone marrow endothelial progenitor cells (BEPCs) have the potential to differentiate into corneal endothelial cells (CECs). Induced BEPCs were revealed to resemble CECs regarding cell shape, expression levels of aquaporin-1 and exhibition of tightly opposed cell junctions. When transplanted into corneas with defective CEC, induced BEPCs have been previously demonstrated to maintain corneal transparency. The results of the previous study suggest that BEPCs have the potential to be used for tissue-engineered corneal endothelium.

The aim of the present study was to observe the morphology, adherence, proliferation and stem cell markers of BEPCs cultured on two hybrid scaffolds: Gelatin/PCL (70:30) and collagen/PCL (70:30). This was performed to investigate the potential value of nanofibrous structures in the culturing of BEPCs and subsequently whether these structures may be used for tissue-engineered corneal endothelium.

\section{Materials and methods}

Preparation of gelatin/PCL and collagen/PCL scaffolds. A gelatin/PCL solution was established in accordance with a previous study (22), gelatin type A (Sigma-Aldrich; Merck KGaA, Darmstadt, Germany) and poly ( $\varepsilon$-caprolactone) PCL (molecular weight, 80,000; Sigma-Aldrich; Merck KGaA), with a mass ratio of $70: 30$, were dissolved in $10 \%(\mathrm{w} / \mathrm{v})$ 2,2,2-trifluoroethanol (TFE; purity $\geq 99 \%$; Sigma-Aldrich; Merck KGaA) under sufficient stirring at room temperature for $24 \mathrm{~h}$. Acetic acid ( $0.2 \%$ diluted in TFE) was added to the solutions to establish a transparent solution. To prepare the collagen/PCL solution, Type I collagen (Sichuan Mingrang Bio-Tech Co., Ltd., Chengdu, China) and PCL with a mass ratio of 70:30 were dissolved in hexafluoroisopropanol (purity $\geq 99 \%$; Sigma Aldrich; Merck KGaA) at a concentration of $8 \%(\mathrm{w} / \mathrm{v})$ and stirred vigorously at room temperature for $48 \mathrm{~h}$. The two solutions were subsequently administered into a $10 \mathrm{ml}$ plastic syringe and subjected to electrospinning using the parameters presented in Table I. A KDS100 syringe pump (KD Scientific, Inc., Holliston, MA, USA) and a high voltage power supply (TXR1020N30-30; Teslaman Technology, Co., Ltd., Dalian, China) were used to regulate the solution dispensing rate and applied voltage, respectively. The obtained membranes were placed in a vacuum oven for 1 week to remove the residual solvent prior to further experimentation.

Morphology and hydrophilicity of scaffolds. The morphologies of the electrospun gelatin/PCL fibers and collagen/PCL fibers were investigated via scanning electron microscopy (SEM; JEOL JSM-5600LV Series Scanning Electron Microscope; Japan Electron Optics Laboratory Co., Ltd., Tokyo, Japan) at an acceleration voltage of 8-10 kV. Electrospun gelatin/PCL fibers and collagen/PCL fibers were fixed with $2.5 \%$ glutaraldehyde for $24 \mathrm{~h}$ at $4^{\circ} \mathrm{C}$. Prior to imaging, samples were coated with gold for $50 \mathrm{sec}$ to increase conductivity. The average diameters of the fibers were determined from the SEM-derived images using Image J $1.40 \mathrm{G}$ software (National Institutes of Health, Bethesda, MD, USA). In addition, a minimum of 100 nanofibers from each sample obtained from different SEM images were manually investigated and analyzed.

The hydrophilicities of the electrospun gelatin/PCL and collagen/PCL fibrous membranes were determined following the analysis of the contact angles of water droplets on membranes, which were measured using a video contact angle instrument (Attension Theta; BiolinScientific, Q-Sense AB, Gothenburg, Sweden). Deionized water (3 $\mu \mathrm{l})$ was automatically dropped onto the surface of the membranes and the contact angle was then determined.

Isolation and culture of BEPCs. Lower-limb bone marrow was obtained a total of 20 female Sprague Dawley rats (age, 4 weeks; weight, $100-120 \mathrm{~g}$ ), which were purchased from the Suzhou Amufi Biological Science and Technology Co., Ltd (Suzhou, China). Rats were bred in a specific-pathogen-free environment with free access to water and fodder. The temperature was kept constant at $25^{\circ} \mathrm{C}$, with a humidity of $\sim 70 \%$ and a $12 \mathrm{~h}$ light/dark cycle. All experimental procedures were approved by the Animal Research Committee of Ninth People's Hospital, Shanghai Jiao Tong University School of Medicine (Shanghai, China). Marrow was isolated by cutting off both ends of the bone, then separated with a syringe. The marrow was washed with phosphate-buffered saline (PBS; Sigma-Aldrich; Merck KGaA). In order to isolate mononuclear cells, myeloid tissue was treated with $3 \mathrm{ml}$ red blood cell lysis buffer (ammonium chloride solution; Stemcell Technologies, Inc., Vancouver, BC, Canada) for $1 \mathrm{~min}$ and then mixed with $7 \mathrm{ml}$ PBS. Following centrifugation at $400 \mathrm{x} \mathrm{g}$ for $10 \mathrm{~min}$ 
Table I. Parameters used for electrospinning fibrous membrane.

\begin{tabular}{|c|c|c|c|c|c|c|c|}
\hline Sample & Solvent & Concentration (\%) & $\begin{array}{c}\text { Applied } \\
\text { voltage }(\mathrm{kV})\end{array}$ & $\begin{array}{l}\text { Feed rate } \\
(\mathrm{ml} / \mathrm{h})\end{array}$ & $\begin{array}{c}\text { Collecting } \\
\text { distance }(\mathrm{cm})\end{array}$ & Temperature & Humidity (\%) \\
\hline $\begin{array}{l}\text { Gelatin/PCL } \\
(70: 30)\end{array}$ & TFE & $10 \mathrm{w} / \mathrm{v}$ & $9-10$ & 1.5 & 13 & $20-25^{\circ} \mathrm{C}$ & $45-65$ \\
\hline $\begin{array}{l}\text { Collagen/PCL } \\
(70: 30)\end{array}$ & HFIP & $8 \mathrm{w} / \mathrm{v}$ & $13-14$ & 1 & 13 & $20-25^{\circ} \mathrm{C}$ & $45-65$ \\
\hline
\end{tabular}

HFIP, hexafluoroisopropanol; TFE, 2,2,2-trifluoroethanol; PCL, polycaprolactone.

at room temperature, cells were suspended in Endothelial Cell Growth Medium-2 (EGM-2; CC-3162; Lonza Group, Ltd., Basel, Switzerland), cultured on $10 \mathrm{~cm}$ culture dishes (Corning Incorporated, Corning, NY, USA) at $37^{\circ} \mathrm{C}$ in $5 \%$ $\mathrm{CO}_{2}$ humidified atmosphere, and subsequently supplemented with hydrocortisone, vascular endothelial growth factor, ascorbic acid, gentamicin, amphotericin-B, recombinant insulin growth factor, human epidermal growth factor, human fibroblast growth factor-B and 5\% fetal bovine serum (FBS; Gibco; Thermo Fisher Scientific, Inc., Waltham, MA, USA). Culturing media was replaced with fresh media a total of 2 days post-seeding. Following this, media was replaced every other day. When cells reached a confluence of $80-90 \%$, BEPCs were treated with $0.25 \%$ trypsin and $0.02 \%$ EDTA (Sigma-Aldrich; Merck KGaA) and were recultured in a different $10 \mathrm{~cm}$ culture dish at a ratio of 1:2. Following this, the collagen/PCL and gelatin/PCL scaffolds were soaked in $75 \%$ ethanol for $10 \mathrm{~min}$. The scaffolds were washed three times with PBS for $15 \mathrm{~min}$ prior to further experimentation.

Morphology and adherence of BEPCs on scaffolds. BEPCs were seeded onto the two scaffolds in a 24-well plate at a density of $2 \times 10^{4}$ cells/well. At day 1 post-seeding, the morphology of BEPCs was investigated via SEM as aforementioned. The adherence of BEPCs cultured on the scaffolds was visualized using rhodamin-phalloidin (1:500; cat. no. 40734ES75; Yeasen, Shanghai, China). The F-actin cytoskeleton was visualized using rhodamin-phalloidin to observe cellular adherence via fluorescence staining. BEPCs that had been cultured for $24 \mathrm{~h}$ were washed with PBS a total of three times for $10 \mathrm{~min}$. BEPCs were fixed with $4 \%$ paraformaldehyde (Sigma-Aldrich; Merck KGaA) for $10 \mathrm{~min}$ at room temperature. Following $10 \mathrm{~min}$ of washing with PBS three times, BEPCs were permeabilized with $0.3 \%$ Triton X-100 (Sigma-Aldrich; Merck KGaA) in PBS. Subsequently, the cells were washed three times with PBS for $10 \mathrm{~min}$, and $200 \mu 1$ Tetramethylrhodamine B isothiocyanate Phalloidin (1:500; cat. no. 40734ES75; Yeasen) was added to each slide. Following $30 \mathrm{~min}$ of incubation under light protection at room temperature, BEPCs were washed for $5 \mathrm{~min}$ for a total of three times. Cell nuclei were then counterstained with 4, 6-diamidino-2-phenylindole (DAPI; Invitrogen; Thermo Fisher Scientific, Inc.). Fluorescent images were subsequently captured using a Nikon eclipse 80i fluorescence microscope at a magnification of $\mathrm{x} 3$ (Nikon Corporation; Tokyo, Japan).
Cell viability and proliferation assays. Viability staining was performed using a calcein acetoxymethyl ester/ethidium homodimer 1 assay (LIVE/DEAD Viability/Cytotoxicity Kit; cat. no. L3224; Thermo Fisher Scientific, Inc.). BEPCs were seeded onto the scaffolds on glass slides in a 24-well plate at a density of $5 \times 10^{4}$ cells/well. A total of $48 \mathrm{~h}$ post-seeding, the cells were incubated in PBS containing $2 \mu \mathrm{l}$ calcein acetoxymethyl ester and $4 \mu \mathrm{l}$ ethidium homodimer 1 at $37^{\circ} \mathrm{C}$ for $45 \mathrm{~min}$. Subsequently, the BEPCs were washed twice with PBS, and fluorescent images were captured using a Nikon eclipse 80i fluorescence microscope at a magnification of x3.

Cell proliferation on the scaffolds was determined to investigate the biocompatibility of the scaffolds. Briefly, BEPCs were seeded onto glass slides and scaffold membranes, as well as the size of slides and membranes, were matched with the 24 well plates. In each well, cells $\left(5 \times 10^{4}\right)$ were seeded in $1 \mathrm{ml}$ of EGM-2 with 5\% FBS. A Cell Count-8 kit (CCK-8; Life, New York, USA) was used to determine cell proliferation, which was performed in accordance with the manufacturer's protocol. Subsequently, the plates were examined with ELISA at a wavelength of $490 \mathrm{~nm}$. At 0, 24, 48 and $72 \mathrm{~h}$ time intervals, the optical density (OD) values from triplicates of each group were determined.

Immunocytochemistry of Ki-67 protein. BEPCs cultured on the two hybrid scaffolds and glass slides were fixed with $4 \%$ paraformaldehyde for $10 \mathrm{~min}$ at room temperature and then permeabilized with $0.3 \%$ Triton X-100 in PBS. Blocking was performed using 10\% normal goat serum (Gibco; Thermo Fisher Scientific, Inc.). Cells were incubated with rabbit monoclonal anti-Ki-67 antibodies (1:200; Abcam, Cambridge, $\mathrm{UK})$ at $4^{\circ} \mathrm{C}$ overnight. Following incubation, the BEPCs were washed with PBS for $10 \mathrm{~min}$ in triplicate, then incubated with fluorescent labeled secondary antibodies (1:500; Alexa Fluor 546 goat anti-rabbit immunoglobulin G; Invitrogen; Thermo Fisher Scientific, Inc.) in PBS for $1 \mathrm{~h}$ at room temperature. Following three washing steps (with PBS), cell nuclei were counterstained with DAPI for $5 \mathrm{~min}$ at room temperature (Invitrogen; Thermo Fisher Scientific, Inc.). Fluorescent images were captured using a Nikon eclipse 80i fluorescence microscope. Ki-67 protein staining was investigated in five visual fields at a magnification of $10 \times 0.30$, and the total percentages of positive cells were calculated.

Immunocytochemistry staining for incorporation of bromodeoxyuridine (BrdU). BEPCs were cultured for $8 \mathrm{~h}$ 
Table II. Primers utilized for reverse transcription-quantitative polymerase chain reaction.

\begin{tabular}{llllr}
\hline Genes & Accession number & \multicolumn{1}{c}{ Forward sequence (5'-3') } & Reverse sequence (5'-3') & $\begin{array}{r}\text { Product } \\
\text { size (bp) }\end{array}$ \\
\hline AQP-1 & NM_012778.1 & ACCTGCTGGCCATTGACTAC & AGGGCACTCCCAATGAATGG & 127 \\
COL8A1 & NM_001107100.1 & TTGCTTACCATGTTCACTGCAAGG & AAAGCCCTTCTTGTACTCGTCGTA & 101 \\
ABCG2 & NM_181381.2 & GTGTAGGTCGGTGTGCGAGT & GATCTATGCCTTTCTAGCTGTCCC & 70 \\
LGR5 & NM_001106784.1 & GCGTCTTCACCTCCTACCTG & TTTCCAGCAAGACGCAACTC & 106 \\
CD166 & NM_031753.1 & AGGGGACAACATCACCCTTC & AGCCTGCCCTGGTAAGTAAA & 82 \\
Caspase-3 & NM_012922.2 & GACCATGGAGAACAACAAAAC & GGCAGGCCTGAATGATGAAG & 494 \\
IL-1 & NM_017019.1 & TCGGGAGGAGACGACTCTAA & GAAAGCTGCGGATGTGAAGT & 201 \\
GAPDH & NM_017008.4 & CATGTTTGTGATGGGTGTGAACCA & AAAGTTGTCATGGATGACCTTGGC & 115 \\
\hline
\end{tabular}

AQP-1, aquaporin 1; COL8A1, collagen type VIII alpha 1; ABCG2, ATP binding cassette subfamily G member 2, LGR5, leucine rich repeat containing G protein-coupled receptor 5; IL-1, interleukin 1.

in the presence of $1 \mu \mathrm{l}$ BrdU (Sigma-Aldrich; Merck KGaA) dissolved in $1 \mathrm{ml}$ culture medium. The cells were fixed in $4 \%$ paraformaldehyde for $15 \mathrm{~min}$ at room temperature and subsequently incubated with $10 \%$ sheep serum (Gibco; Thermo Fisher Scientific, Inc.) and $0.25 \%$ Triton X-100 in PBS for $1 \mathrm{~h}$ at room temperature. Following incubation, the BEPCs were washed in PBS, incubated with $0.83 \% \mathrm{HCl}$ for $30 \mathrm{~min}$ at room temperature, washed in Hanks' Balanced Salt Solution and PBS at room temperature. Following incubation with anti-BrdU antibodies (1:800; cat. no. ab8152; Abcam) at $4^{\circ} \mathrm{C}$ overnight, BEPCs were washed in PBS and incubated with fluorescent-conjugated secondary antibodies (1:500; Alexa Fluor 546-goat anti-rabbit) for $1 \mathrm{~h}$ at room temperature. Cell nuclei were subsequently stained with DAPI for $5 \mathrm{~min}$ at room temperature. Fluorescent images were obtained using a Nikon eclipse 80i fluorescence microscope. BrdU protein staining was investigated in five visual fields at a magnification of 10x0.30, and the total percentages of live and positive cells were subsequently determined.

Investigation of stem cell markers. The identification and characterization of BEPCs were performed using rabbit monoclonal anti-CD34antibodies (1:200; Abcam) and rabbit monoclonal anti-CD133antibodies (1:200; Abcam). Following culturing of BEPCs on scaffolds and glass slides for 3 days, BEPCs were fixed with $4 \%$ paraformaldehyde, dissolved in PBS for $10 \mathrm{~min}$ and permeabilized using $0.3 \%$ Triton $\mathrm{X}-100$ in PBS. BEPCs were then subjected to treatment with $10 \%$ normal goat serum (Gibco; Thermo Fisher Scientific, Inc.). Following this, cells were incubated with rabbit monoclonal anti-CD34 and rabbit monoclonal anti-CD133 antibodies at $4^{\circ} \mathrm{C}$ for $12 \mathrm{~h}$. The coverslips and two hybrid scaffolds were subsequently washed three times with PBS for 10 min each. The two hybrid groups and the control group were incubated with fluorescent labeled secondary antibodies (1:500; Alexa Fluor 546 goat anti-rabbit immunoglobulin G; BD Biosciences, Franklin Lakes, NJ, USA) in PBS for $1 \mathrm{~h}$. Following three washes with PBS, cell nuclei were counterstained with DAPI for $5 \mathrm{~min}$ at room temperature. Fluorescent images were obtained using a Nikon eclipse 80i fluorescence microscope. CD34 protein staining was investigated in five visual fields at a magnification of $10 \times 0.30$, and the total percentages of live and positive cells were determined.

Reverse transcription-quantitative polymerase chain reaction (RT-qPCR). Gene expression levels of BEPCs cultured on two hybrid scaffolds and glass slides were analyzed via RT-qPCR. mRNA was isolated from samples at the 7 day time interval using TRIzol reagent (Invitrogen; Thermo Fisher Scientific, Inc.). A NanoDrop ND1000 Spectrophotometer (Thermo Fisher Scientific, Inc.) was used to quantify the extracted RNA samples. Samples with A260/A280 ratios between 1.9-2.1 were used for subsequent analysis. Total RNA was subjected to RT using the PrimeScript RT reagent kit (Perfect Real Time; Takara Biotechnology Co., Ltd., Dalian, China). RT-qPCR was performed in a $10 \mu \mathrm{l}$ reaction volume containing $1 \mu \mathrm{l}$ of complementary DNA, $3 \mu \mathrm{l}$ of doubled-distilled $\mathrm{H}_{2} \mathrm{O}, 5 \mu \mathrm{l}$ of reaction mixture and $1 \mu \mathrm{l}$ of primers. The primer sequences used are presented in Table II. In the present study, seven primers [aquaporin 1 (AQP1), collagen type VIII alpha 1 (COL8A1), ATP binding cassette subfamily G member 2 (ABCG2), leucine rich repeat containing $G$ protein-coupled receptor 5 (LGR5), Apoptosis-Related Cysteine Peptidase (Caspase-3), Activated Leukocyte Cell Adhesion Molecule (CD166) and interleukin 1 (IL-1)] were used to investigate proliferation, stem cell characteristics, apoptosis and inflammatory reaction of BEPCs in the scaffold groups and the control group. RT-qPCR was performed using a Light Cycler 96 System (Roche Diagnostics, Basel, Switzerland). Each assay was performed in triplicate. Relative gene expression was analyzed by using the Pfaffl method (23).

Statistical analysis. Data in the present study were presented as the mean \pm standard error of the mean. One-way analysis of variance with Tukey's HSD post hoc test were used to investigate the statistical significance between groups. $\mathrm{P}<0.05$ was considered to indicate a statistically significant difference.

\section{Results}

Morphology of scaffolds. The fiber morphology and dimension distribution of the two variations of electrospun membranes 

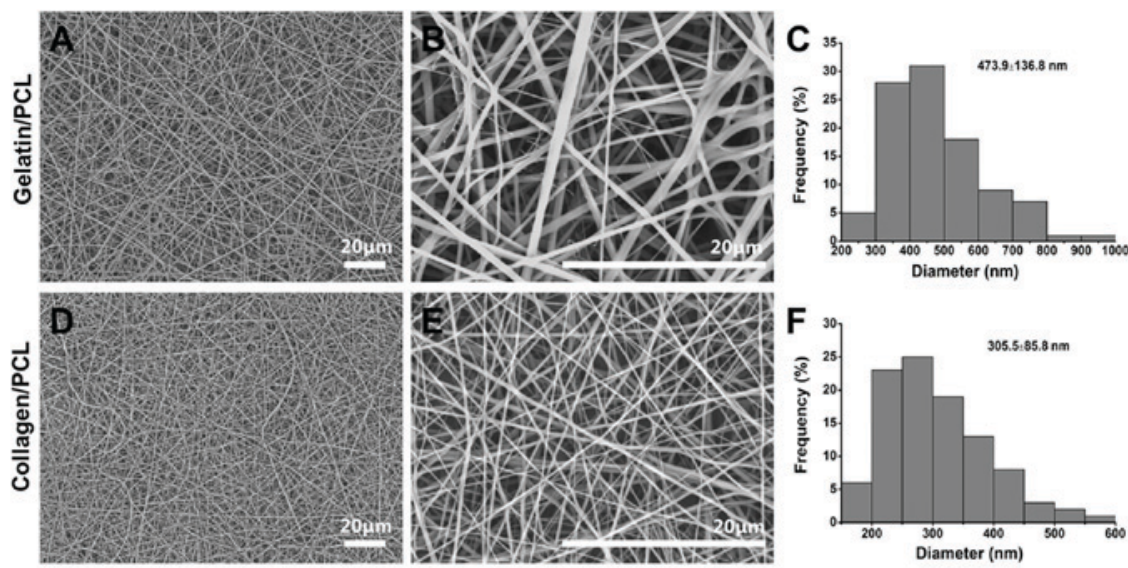

G

Morphology of Scaffolds
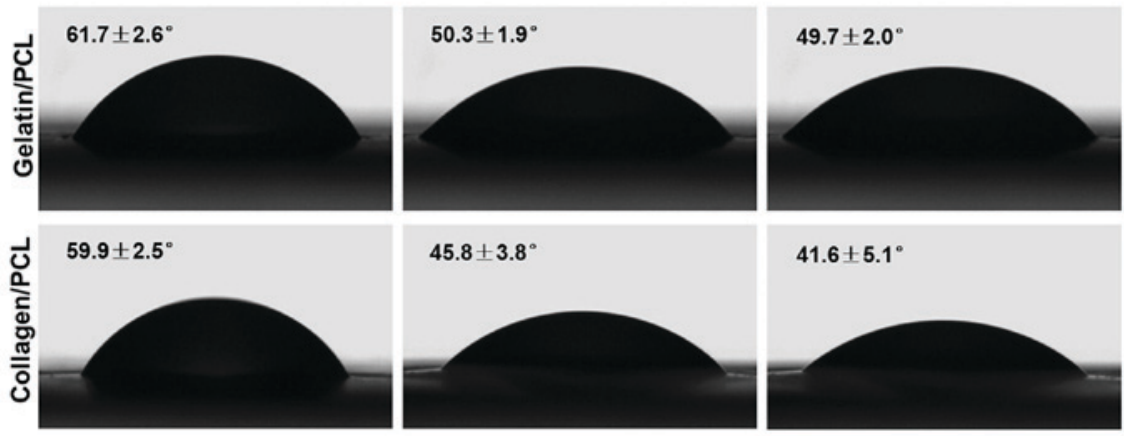

$10 \mathrm{sec}$

$0 \mathrm{sec}$

Water Contact Angle of Scaffolds

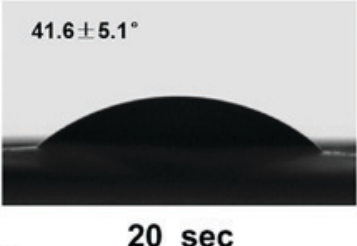

$20 \mathrm{sec}$

Figure 1. (A-G) SEM and water contact angles of gelatin/PCL and collagen/PCL electrospun fibrous membranes. (A and B) Gelatin/PCL, (D and E) collagen/PCL and diameter distributions of (C) gelatin/PCL and (F) collagen/PCL membranes. Scale bars, $20 \mu \mathrm{m}$. (G) Water contact angles of two hybrid scaffolds. SEM, scanning electron microscope; PCL, polycaprolactone; SEM, scanning electron microscopy.

were investigated via SEM. As presented in Fig. 1A-C, gelatin/PCL (70:30) fibers were demonstrated to be smooth and fine, with an average fiber diameter of $473.9 \pm 136.8 \mathrm{~nm}$. Electrospun collagen/PCL fibrous membranes (70:30) were also revealed to be smooth and uniform, with an average diameter of $305.5 \pm 85.8 \mathrm{~nm}$ (Fig. 1D-F). According to the results, the two samples presented with similar morphology and microstructures despite varying materials and electrospinning conditions.

Water contact angle of scaffolds. The hydrophilicity of the membranes, which can be altered according to its composition, has marked effects on the adhesion, proliferation and viability of cells (24). The water contact angle may be determined to investigate wettability. As revealed in Fig. 1G, water droplets were rapidly absorbed into the fibrous networks at the beginning, the contact angle reached almost the same as that at $0 \mathrm{sec}$ $\left(61.7 \pm 2.6^{\circ}\right.$ vs. $\left.59.9 \pm 2.5^{\circ}\right)$. As the time increased, the exhibited contact angle slowly decreased. Notably, the collagen/PCL (70:30) fibrous membrane decreased at a faster rate compared with the gelatin/PCL (70:30) fibrous membrane. These results suggested that the gelatin/PCL and collagen/PCL electrospun membranes were hydrophilic, and thus potentially suitable for cell adhesion.

Cell morphology and adherence on scaffolds. SEM was used to investigate cell morphology and adherence on scaffolds.
Following 24 h of cell seeding, BEPCs were revealed to be well spread on and strongly adhered to both scaffolds. BEPCs on the two hybrid scaffolds exhibited a three-dimensional interdigitated structure and presented a crossover growth trend. The results of SEM were indicated (Fig. 2A). To investigate the adherence of BEPCs and the cellular compatibility on the two hybrid scaffolds, F-actin cytoskeletons were visualized in the scaffold groups and the control group using rhodamin-phalloidin. Cells in the hybrid scaffold groups exhibited aggregation and increased levels of F-actin bundles compared with cells in the control group (Fig. 2B). These results suggested that collagen/PCL (70:30) and gelatin/PCL (70:30) scaffolds exhibited cellular compatibility and benefited cell adherence.

Cell proliferation and viability on hybrid scaffolds. A live\&dead Viability/Cytotoxicity kit was used to investigate the toxicity of the materials. Apart from the results regarding cell densities, no significant differences were exhibited between any of the groups. Marked levels of dead cells were not revealed in any groups (Fig. 3A). Immunocytochemistry staining of $\mathrm{Ki}-67$ and BrdU was performed to investigate levels of cell proliferation. The results of Ki-67 and BrdU staining demonstrated that BEPCs on collagen/PCL (70:30) and gelatin/PCL (70:30) exhibited greater levels of proliferation compared with the control group (Fig. 3B and C)., $\mathrm{D}$ and E, compared with the control group, both scaffold 

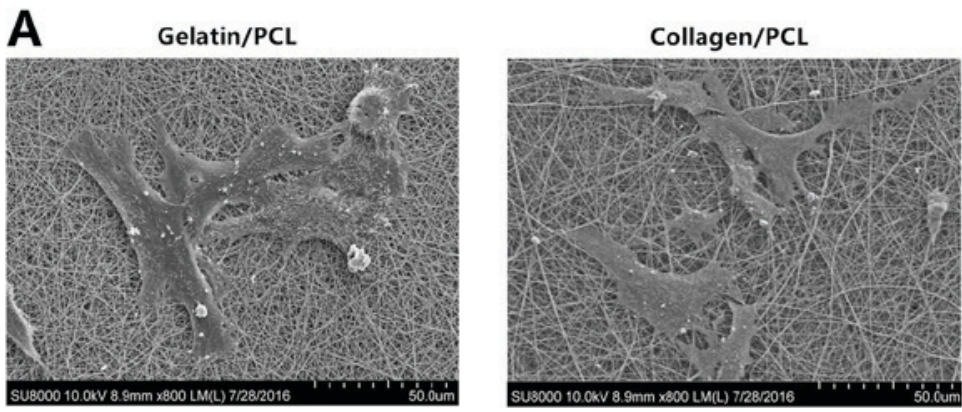

\section{Scanning Electron Microscope}

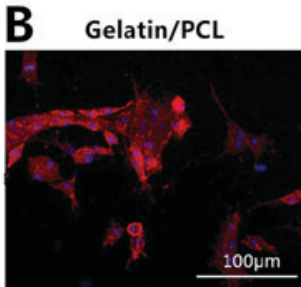

Collagen/PCL

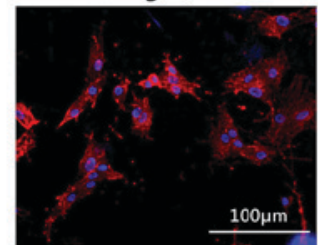

F-actin Cytoskeleton

Figure 2. Morphology and adherence of BEPCs to scaffolds. (A) BEPCs were revealed to be strongly adhered and well spread on the two scaffolds a total of $24 \mathrm{~h}$ post-seeding (scale bar, $50 \mu \mathrm{m}$ ). (B) The F-actin cytoskeleton was visualized via rhodamin-phalloidin staining (scale bar, $100 \mu \mathrm{m}$ ). PCL, polycaprolactone; BEPCs, bone marrow endothelial progenitor cells.
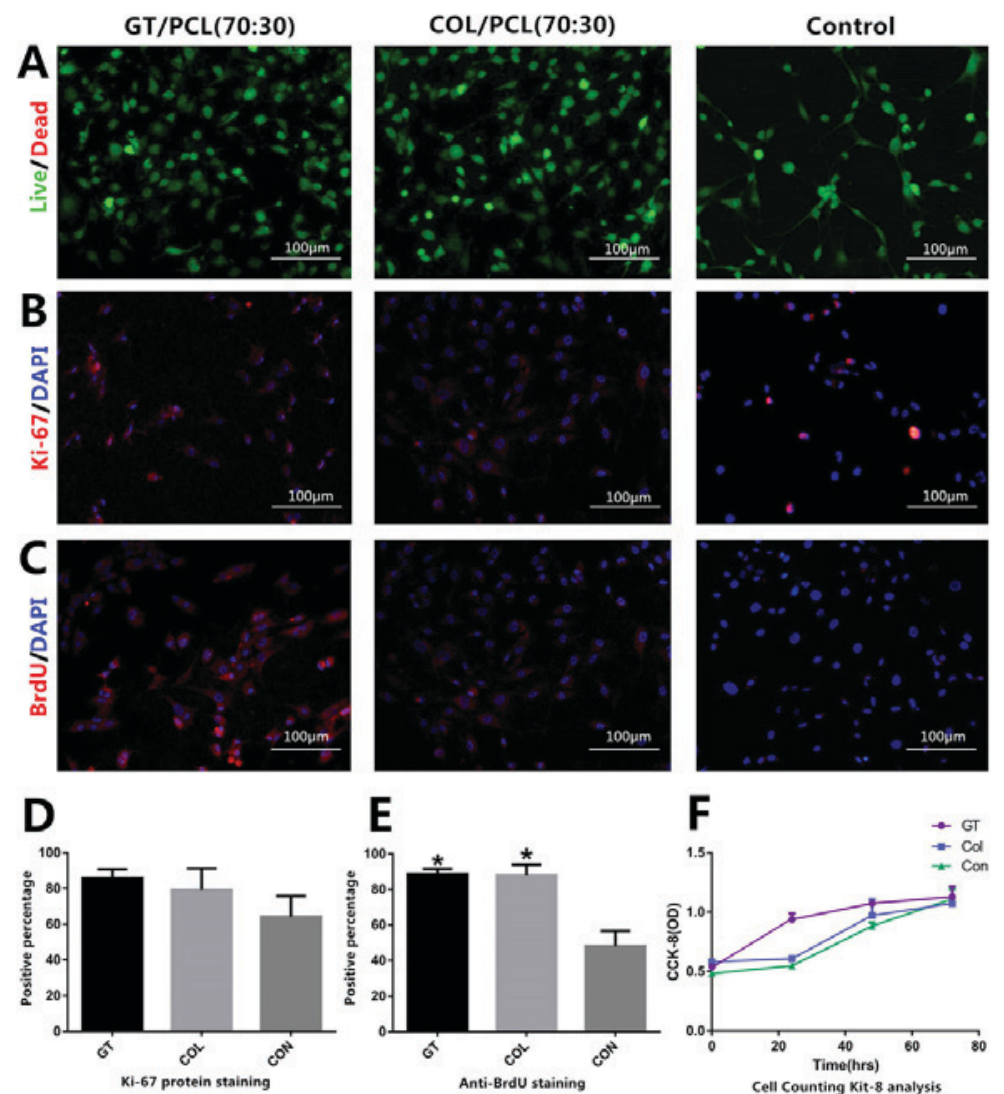

Figure 3. Proliferation and viability of BEPCs on scaffolds. (A) Results of the live\&dead kit test. Expression levels of (B) Ki-67 and (C) BrdU proteins in BEPCs were investigated (scale bar, $100 \mu \mathrm{m}$ ). Histograms revealed the percentage of positive staining of (D) Ki-67 and (E) BrdU proteins. (F) Proliferation of BEPCs cultured on the two hybrid scaffolds groups and the control group were determined using a Cell Counting Kit-8 kit. "P<0.05 vs. controls. DAPI, 4,6-diamidino-2-phenylindole; PCL, polycaprolactone; BEPCs, bone marrow endothelial progenitor cells; BrdU, bromodeoxyuridine.

groups presented a statistically significant increase following BrdU staining $(\mathrm{P}<0.05$; Fig. 3E). The results of Ki-67 and
BrdU staining, which was investigated in five visual fields at a magnification of $10 \times 0.30$, are presented in Fig. 3D and E. 

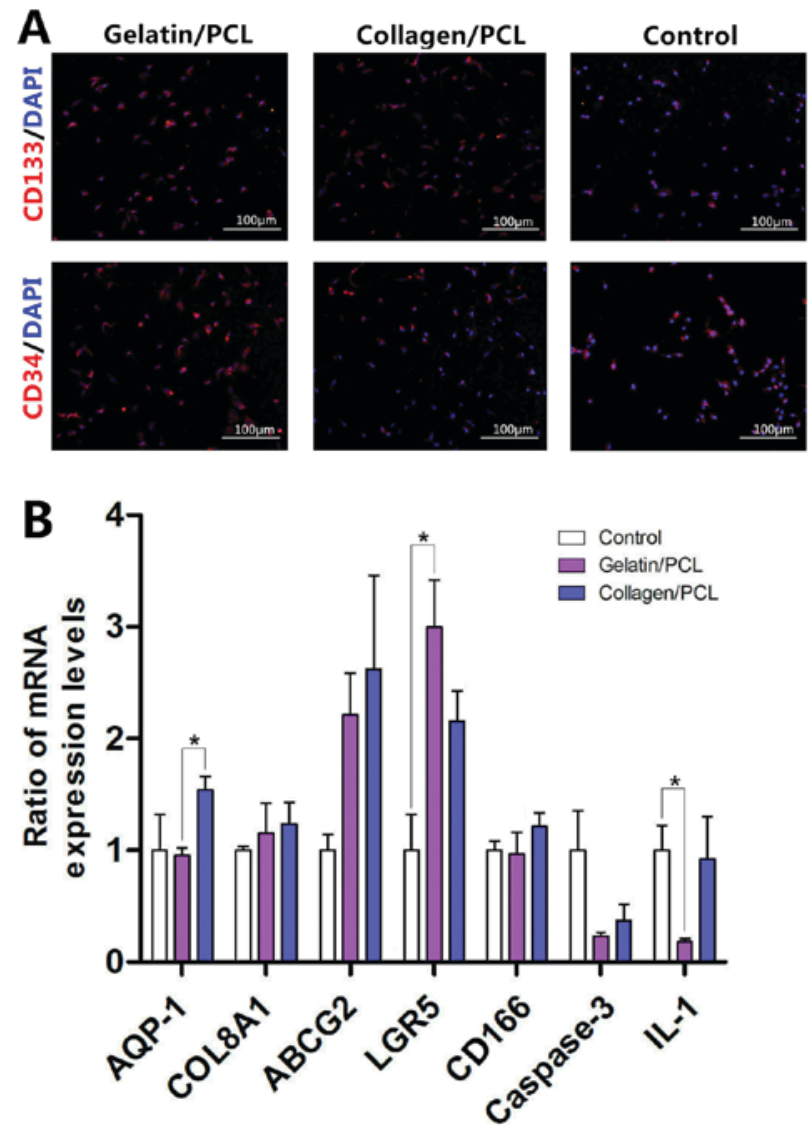

Figure 4. Expression levels of stem cell markers exhibited by BEPCs on hybrid scaffolds. Protein staining revealing the expression levels of (A) CD133 and (B) CD34 (scale bar, $100 \mu \mathrm{m}$ ). (B) Gene expression levels of scaffold groups and the control group. " $\mathrm{P}<0.05$ vs. controls. CD, cluster of differentiation; BEPCs, bone marrow endothelial progenitor cells; AQP-1, aquaporin 1; COL8A1, collagen type VIII alpha 1; ABCG2, ATP binding cassette subfamily G member 2, LGR5, leucine rich repeat containing G protein-coupled receptor 5; IL-1, interleukin 1.

Furthermore, CCK-8 analysis was performed to investigate the cell proliferation and cellular compatibility of the two hybrid scaffolds. The results demonstrated that the OD values were not significantly different between the collagen/PCL (70:30) group and the control group. Notably, the OD values exhibited by the gelatin/PC L (70:30) group were increased compared with the two groups at 0,24 and $48 \mathrm{~h}$ time intervals. At the $72 \mathrm{~h}$ time interval, the OD values were not significantly different among the three groups (Fig. 3F).

Stem cell characteristics of BEPCs on two hybrid scaffolds. Immunocytochemistry analysis investigating the expression levels of CD133 and CD34 proteins, which represent biomarkers of stem cells, was performed to determine and evaluate the stem cell characteristics of BEPCs on two hybrid scaffolds. Cultured BEPCs on the two hybrid scaffolds demonstrated increased levels of fluorescence intensity exhibited by the CD133 and CD34 proteins compared with the control group; however, this result was not statistically significant (Fig. 4A). In addition, both scaffold groups exhibited increased expression levels of ABCG2 and LGR5 compared with the control group. Notably, the expression levels of other proteins (AQP-1, COL8A1 and CD166) on both scaffold groups were not statistically different compared with the control group (Fig. 4B).
Biocompatibility of two hybrid scaffolds. The expression levels of caspase-3 and IL-1 were investigated in the present study to determine the biocompatibility of the two hybrid scaffolds. Apoptosis levels, as determined via the investigation of caspase-3 expression levels, were revealed to be decreased in the two scaffold groups compared with the control group; however, this result was not statistically significant. Furthermore, the results demonstrated that there were no significant differences in the expression levels of IL-1 exhibited in the collagen/PCL group and control group; whereas the expression levels of IL-1 exhibited by the gelatin/PCL group were significantly suppressed compared with the control group (Fig. 4B). These results suggested that the two scaffolds exhibited biocompatibility.

\section{Discussion}

Tissue-engineering for corneal endothelium has been heavily researched in recent years regarding the treatment for patients with corneal endothelial decompensation. Establishing a suitable carrier material is important for establishing tissue-engineered endothelium. Natural polymers, such as collagen and gelatin, have previously been electrospun into fibrous scaffolds for tissue engineering of corneal epithelium (10). However, it remains unknown whether collagen and gelatin are appropriate materials for tissue-engineered 
endothelium. Scaffolds used in tissue-engineered corneal endothelium are required to exhibit sufficient mechanical strength and good biocompatibility, which may promote cell proliferation and differentiation. Hybrid scaffolds could meet the requirements by taking advantage of natural and synthetic material properties $(25,26)$. In the present study, gelatin/PCL and collagen/PCL hybrid scaffolds were successfully established using electrospun techniques, and the modulating responses of BEPCs cultured on the scaffolds were investigated. This aimed to elucidate the potential applicability of the scaffolds in tissue-engineered endothelium.

The two hybrid scaffolds established in the present study demonstrated expected hydrophilicity, wettability and biocompatibility characteristics, which were most likely due to the exhibition of beneficial characteristics associated with natural and synthetic polymers. SEM analyses demonstrated that BEPCs presented a crossover growth trend and exhibited a three-dimensional interdigitated structure on the two scaffolds. As presented in Fig. 2, BEPCs in the two hybrid scaffold groups exhibited increased levels of aggregation and F-actin bundles compared with the control group. The cellular morphology and adhesion suggested that the electrospun nanofibers established in the present study successfully emulated the properties of the extracellular matrix. BEPCs were well spread and strongly adhered to both scaffolds.

BEPCs cultured on both scaffolds exhibited greater levels of proliferation and viability. Immunocytochemistry staining of Ki-67 and BrdU proteins revealed that the two scaffolds provided a suitable environment for cell growth, likely due to properties exhibited by the scaffolds that are associated with the extracellular matrix. However, CCK-8 analysis demonstrated that there were no significant differences in the proliferation levels exhibited by each group. At 24 and $48 \mathrm{~h}$ time intervals, the OD values of gelatin/PCL were increased compared with the other two groups, whereas the collagen/PCL group did not exhibit significant differences in OD values compared with the control group. In addition, the OD values exhibited at 0 and $72 \mathrm{~h}$ time intervals were not significantly different between any of the groups. Therefore, it may be suggested that such differences may be due to dissimilar topography between scaffolds and glass slides, thus permitting cells to attach and spread more easily from the outset. The growth of BEPCs on scaffolds for increased durations of time requires further study. In the present study, few dead cells on scaffolds were observed, which suggested that the two hybrid nanofiber scaffolds investigated in the present study were safe and nontoxic for proliferating cells. Furthermore, the results revealed that the mRNA expression levels of caspase- 3 and IL-1 were decreased in gelatin/PCL groups, which suggested that the scaffold did not induce inflammatory responses or apoptosis. The collagen/PCL scaffold exhibited decreased levels of caspase-3, while the expression levels of IL-1 did not demonstrate a significant difference compared with the control group. A previous study revealed that, when cultured with NIH 3T3 cells, collagen exhibits significantly enhanced levels of cytocompatibility compared with gelatin (27). In the present study, no significant differences between the two hybrid scaffolds were demonstrated, which suggests that the two natural components may be safely used when applied to the culturing BEPCs. The biocompatibility of scaffolds used in tissue-engineered endothelium is important to ensure cell density and to forma cell monolayer (28-30).

Tissue-engineered endothelium requires a substratum with an organized monolayer of cells. The morphology and cell density of the newly formed cell monolayer depends on the differentiation status of the transplanted cells (28-31). It is important for scaffolds to enable cells to retain characteristics associated with stem cells. BEPCs cultured on hybrid nanofibers scaffolds demonstrated a marked increase in the levels of stem cell markers. CD34 and CD133 protein fluorescence intensities expressed by the scaffold groups were enhanced compared with the control group; however, this result was not statistically significant. This suggested that BEPCs cultured on the two hybrid scaffolds demonstrated more avidity. PCR analyses of marker genes and differentiation genes associated with stem cells, including ABCG 2 and LGR5 (32-34), exhibited increased expression levels in the scaffold groups compared with the control group. Nanofibers were revealed to simulate the three-dimensional extracellular matrix, and thus it may be suggested that approximate three-dimensional topography and chemical stimulation of hybrid nanofiber scaffolds have important roles in pluripotency maintaining of BEPC. However, expression levels of marker genes associated with CECs in the scaffold groups, including AQP-1, COL8A1 and CD166, were revealed to not be significantly different compared with the control group in the present study. These results suggested that the two scaffolds may enhance the pluripotency of BEPCs via numerous mechanisms; however, culturing on the scaffolds alone did not promote the differentiation of BEPCs into CECs. Whether BEPCs cultured on the scaffolds may differentiate into CECs requires further study.

In the present study, collagen/PCL (70:30) and gelatin/PCL (70:30) combined scaffolds were successfully established using electrospun techniques and exhibited sufficient levels of hydrophilicity, wettability and biocompatibility. BEPCs cultured on the two scaffolds exhibited greater levels of proliferation and adhesion, and retained more characteristics associated with stem cells. The two scaffolds exhibited the possibility of enhancing the differentiation potential of BEPCs. These results suggested that the two hybrid scaffolds maybe used to culture BEPCs that exhibit the potential to differentiate into corneal endothelial-like cells. By improving the manufacturing method of these two hybrid scaffolds, novel strategies to enhance the transparency and mechanical properties of scaffolds so that they are more appropriate for tissue-engineered endothelium may be established. The results of the present study may provide novel ideas for the treatment of corneal endothelial dystrophy.

\section{Acknowledgements}

Not applicable.

\section{Funding}

This study was supported by grants from the National Natural Science Foundation (grant nos. 81370992, 81770332, 81570812, 81500765, 81600774 and 81601622), The Science and Technology Commission of Shanghai 
(grant no. 17DZ2260100), National High Technology Research and Development Program (863 Program; grant no. 2015AA020311), Shanghai Municipal Education Commission Gaofeng Clinical Medicine Grand Support (grant no. 20161421), Youth Science and Technology Talent Sail Plan of Shanghai (grant no. 15YF1407400), Shanghai Municipal Commission of Health and Family Planning Found (grant no. 20144Y0221) and the Shanghai Young Doctor Training Program.

\section{Availability of data and materials}

The datasets used or analyzed during the current study are available from the corresponding author on reasonable request.

\section{Authors' contributions}

YH, BF, FL and YF conceived and designed the current study. YH, BF, WZ, CY, FY, QY and CS performed the experiments. YH and CS wrote the paper. BF, FL and YF reviewed and edited the manuscript. All authors read and approved the manuscript.

\section{Ethics approval and consent to participate}

All experimental procedures were approved by the Animal Research Committee of Ninth People's Hospital, Shanghai Jiao Tong University School of Medicine (Shanghai, China).

\section{Patient consent for publication}

Not applicable.

\section{Competing interests}

The authors declare that they have no competing interests.

\section{References}

1. Bourne WM, Nelson LR and Hodge DO: Central corneal endothelial cell changes over a ten-year period. Invest Ophthalmol Vis Sci 38: 779-782, 1997.

2. Peh GS, Beuerman RW, Colman A, Tan DT and Mehta JS Human corneal endothelial cell expansion for corneal endothelium transplantation: An overview. Transplantation 91: 811-819, 2011.

3. Navaratnam J, Utheim TP, Rajasekhar VK and Shahdadfar A: Substrates for expansion of corneal endothelial cells towards bioengineering of human corneal endothelium. J Funct Biomater 6: 917-945, 2015.

4. Joyce NC: Proliferative capacity of the corneal endothelium. Prog Retin Eye Res 22: 359-389, 2003.

5. Proulx S and Brunette I: Methods being developed for preparation, delivery and transplantation of a tissue-engineered corneal endothelium. Exp Eye Res 95: 68-75, 2012.

6. Bashur CA, Dahlgren LA and Goldstein AS: Effect of fiber diameter and orientation on fibroblast morphology and proliferation on electrospun poly(D,L-lactic-co-glycolic acid) meshes. Biomaterials 27: 5681-5688, 2006.

7. Xu C, Inai R, Kotaki M and Ramakrishna S: Electrospun nanofiber fabrication as synthetic extracellular matrix and its potential for vascular tissue engineering. Tissue Eng 10 1160-1168, 2004.

8. Li WJ, Laurencin CT, Caterson EJ, Tuan RS and Ko FK: Electrospun nanofibrous structure: A novel scaffold for tissue engineering. J Biomed Mater Res 60: 613-621, 2002.
9. Chew SY, Wen J, Yim EK and Leong KW: Sustained release of proteins from electrospun biodegradable fibers. Biomacromolecules 6: 2017-2024, 2005.

10. Liu Y, Ren L and Wang Y: Crosslinked collagen-gelatin-hyaluronic acid biomimetic film for cornea tissue engineering applications. Mater Sci Eng C Mater Biol Appl 33: 196-201, 2013.

11. Wang K, Chen X, Pan Y, Cui Y, Zhou X, Kong D and Zhao Q: Enhanced vascularization in hybrid PCL/gelatin fibrous scaffolds with sustained release of VEGF. Biomed Res Int 2015: 865076, 2015.

12. Hosseini Y, Agah M and Verbridge SS: Endothelial cell sensing, restructuring, and invasion in collagen hydrogel structures. Integr Biol (Camb) 7: 1432-1441, 2015.

13. Ye J, Wang J, Zhu Y, Wei Q, Wang X, Yang J, Tang S, Liu H, Fan J, Zhang F, et al: A thermoresponsive polydiolcitrate-gelatin scaffold and delivery system mediates effective bone formation from BMP9-transduced mesenchymal stem cells. Biomed Mater 11: 025021, 2016.

14. Zhang Y, Ouyang H, Lim CT, Ramakrishna S and Huang ZM: Electrospinning of gelatin fibers and gelatin/PCL composite fibrous scaffolds. J Biomed Mater Res B Appl Biomater 72: 156-165, 2005.

15. Tillman BW, Yazdani SK, Lee SJ, Geary RL, Atala A and Yoo JJ: The in vivo stability of electrospun polycaprolactone-collagen scaffolds in vascular reconstruction. Biomaterials 30: 583-588, 2009.

16. McKenna KA, Hinds MT, Sarao RC, Wu PC, Maslen CL, Glanville RW, Babcock D and Gregory KW: Mechanical property characterization of electrospun recombinant human tropoelastin for vascular graft biomaterials. Acta Biomater 8: 225-233, 2012.

17. Han F, Jia X, Dai D, Yang X, Zhao J, Zhao Y, Fan Y and Yuan X: Performance of a multilayered small-diameter vascular scaffold dual-loaded with VEGF and PDGF. Biomaterials 34: 7302-7313, 2013.

18. Yin A, Zhang K, McClure MJ, Huang C, Wu J, Fang J, Mo X, Bowlin GL, Al-Deyab SS and El-Newehy M: Electrospinning collagen/chitosan/poly(L-lactic acid-co-e-caprolactone) to form a vascular graft: Mechanical and biological characterization. J Biomed Mater Res A 101: 1292-1301, 2013.

19. Chen J, Yan C, Zhu M, Yao Q, Shao C, Lu W, Wang J, Mo X, $\mathrm{Gu}$ P, Fu Y and Fan X: Electrospun nanofibrous SF/P (LLA-CL) membrane: A potential substratum for endothelial keratoplasty. Int J Nanomedicine 10: 3337-3350, 2015.

20. Shao $\mathrm{C}, \mathrm{Fu} \mathrm{Y}, \mathrm{Lu} \mathrm{W}$ and Fan X: Bone marrow-derived endothelial progenitor cells: A promising therapeutic alternative for corneal endothelial dysfunction. Cells Tissues Organs 193: 253-263, 2011.

21. Shao C, Chen J, Chen P, Zhu M, Yao Q, Gu P, Fu Y and Fan X: Targeted transplantation of human umbilical cord blood endothelial progenitor cells with immunomagnetic nanoparticles to repair corneal endothelium defect. Stem Cells Dev 24: 756-767, 2015.

22. Feng B, Tu H, Yuan H,Peng HandZhang Y: Acetic-acid-mediated miscibility toward electrospinning homogeneous composite nanofibers of GT/PCL. Biomacromolecules 13: 3917-3925, 2012.

23. Yoeruek E, Saygili O, Spitzer MS, Tatar O, Bartz-Schmidt KU and Szurman P: Human anterior lens capsule as carrier matrix for cultivated human corneal endothelial cells. Cornea 28: 416-420, 2009.

24. Goddard JM and Joseph JH: Polymer surface modification for the attachment of bioactive compounds. Progr Polymer Sci 32: 698-725, 2007.

25. Duan H, Feng B, Guo X, Wang J, Zhao L, Zhou G, Liu W, Cao Y and Zhang WJ: Engineering of epidermis skin grafts using electrospun nanofibrous gelatin/ polycaprolactone membranes. Int J Nanomedicine 8: 2077-2084, 2013

26. Fu W, Liu Z, Feng B, Hu R, He X, Wang H, Yin M, Huang H, Zhang $\mathrm{H}$ and Wang W: Electrospun gelatin/PCL and collagen/PLCL scaffolds for vascular tissue engineering. Int J Nanomedicine 9: 2335-2344, 2014

27. Wang Y, Zhang W, Yuan J and Shen J: Differences in cytocompatibility between collagen, gelatin and keratin. Mater Sci Eng C Mater Biol Appl 59: 30-34, 2016.

28. Engelmann K, Drexler D and Böhnke M: Transplantation of adult human or porcine corneal endothelial cells onto human recipients in vitro. Part I: Cell culturing and transplantation procedure. Cornea 18: 199-206, 1999. 
29. Engelmann K, Bednarz J and Bohnke M: Endothelial cell transplantation and growth behavior of the human corneal endothelium. Ophthalmologe 96: 555-562, 1999 (In German).

30. Engelmann K, Bohnke M and Friedl P: Isolation and long-term cultivation of human corneal endothelial cells. Invest Ophthalmol Vis Sci 29: 1656-1662, 1988.

31. Tan DT, Anshu A and Mehta JS: Paradigm shifts in corneal transplantation. Ann Acad Med Singapore 38: 332-338, 2009.

32. Hirata-Tominaga K, Nakamura T, Okumura N, Kawasaki S, Kay EP, Barrandon Y, Koizumi N and Kinoshita S: Corneal endothelial cell fate is maintained by LGR5 through the regulation of hedgehog and Wnt pathway. Stem Cells 31: 1396-1407, 2013.
33. Okumura N, Nakamura T, Kay EP, Nakahara M, Kinoshita S and Koizumi N: R-spondin1 regulates cell proliferation of corneal endothelial cells via the Wnt3a/ $\beta$-catenin pathway. Invest Ophthalmol Vis Sci 55: 6861-6869, 2014.

34. Holland JD, Klaus A, Garratt AN and Birchmeier W: Wnt signaling in stem and cancer stem cells. Curr Opin Cell Biol 25: 254-264, 2013. 